\title{
3D Printing Technology for Tapered Optical Fiber Protection With Gas Sensing Possibilities
}

\author{
Kaleb Roncatti de SOUZA, Jonas H. OSÓRIO, Juliana B. CARVALHO, \\ Beatriz Mota LIMA, and Cristiano M. B. CORDEIRO*
}

\author{
Gleb Wataghin Physics Institute, State University of Campinas, Campinas 13083-970, Brazil \\ *Corresponding author: Cristiano M. B. CORDEIRO $\quad$ E-mail: cmbc@ifi.unicamp.br
}

\begin{abstract}
We present a new procedure for protecting micro-optical fibers (tapered fibers) by using the 3-dimensional (3D) printing technology. A standard single-mode optical fiber was tapered down to the diameter of $1 \mu \mathrm{m}$ and embedded in a polymeric matrix obtained by an additive manufacturing routine. We show that the proposed structure protects the fiber taper against environmental humidity while keeping permeability to gas flow and the possibility of the realization of gas detection experiments. To our knowledge, this is the first time 3D printed casings were applied to protect fiber tapers from humidity deterioration. We envisage this new approach will allow the development of new fiber taper devices to better resist in humid environments.
\end{abstract}

Keywords: 3D printing; additive manufacturing; optical fiber; tapered fibers; sensing

Citation: Kaleb Roncatti de SOUZA, Jonas H. OSÓRIO, Juliana B. CARVALHO, Beatriz Mota LIMA, and Cristiano M. B. CORDEIRO, “3D Printing Technology for Tapered Optical Fiber Protection With Gas Sensing Possibilities," Photonic Sensors, 2020, 10(4): 298-305.

\section{Introduction}

Recently, the field of optic fiber sensors has experienced significant development, and nowadays, it consists of a highly active scientific and technological area. Thus, optical fibers have demonstrated themselves to be an excellent platform for obtaining a wide variety of sensors to probe parameters such as temperature, hydrostatic pressure, strain, and refractive index. Key advantages of optical fibers sensors are their inherent electromagnetic immunity, high sensitivity, compactness, and the capability of being used in harsh environments [1].

Several configurations can be used for fiber optics sensing activity. Microstructured optical fibers (optical fibers endowed with special arrangements of air holes which run along the whole fiber length) [2] assume a prominent position due to their vast design versatility, which allows them to have specific and tailorable properties [3]. On the other hand, standard optical fibers (without cross-sectional microstructures) can also be used as sensors if set in specific configurations, such as in SMS (single mode-multimode-single mode) configurations [4], or post-processed (for example, via the utilization of fiber gratings [5] and tapered fibers $[6,7])$.

Fiber tapers are optical fibers that have their dimensions reduced in a controllable fashion. They can be obtained by using fusion splicers [8], ceramic micro-heaters [9], $\mathrm{CO}_{2}$ lasers [10], or by using so-called flame brushing technique [11]. In the latter, an oscillating flame heats a well-defined region of

Received: 15 October 2019 / Revised: 14 May 2020

(C) The Author(s) 2020. This article is published with open access at Springerlink.com

DOI: $10.1007 / \mathrm{s} 13320-020-0592-3$

Article type: Regular 
the fiber while it is controllably stretched. It entails, by mass conservation, an increase in the fiber length and the subsequent reduction of the diameter of the selected region of the fiber [7].

The use of tapered fibers has been demonstrated to be a very efficient route to make standard optical fibers sensitive to the fiber external medium. It is possible because the reduction of the waveguide diameter causes the optical mode evanescent field to permeate the external fiber environment [7]. This property allows the optical mode characteristics to be strongly dependent on the external medium. For example, it opens up the opportunity of tailoring the fiber taper dispersion characteristics by immersing it into fluids to achieve supercontinuum generation [12]. Additionally, this property is highly useful for obtaining sensing devices. Fiber tapers allow, for example, obtaining highly sensitive refractive index sensors for probing liquid samples. In these sensors, sensitivity figures as high as thousands of nanometers per RIU (refractive index unit) can be attained [13]. Moreover, fiber tapers can also be used to probe gaseous samples via the measurement of gas absorption lines [14].

Thin silica fibers can have remarkably high mechanical strength, which can be even greater than those of steel or Kevlar with similar diameters [15]. Nonetheless, they should be protected against humidity and dust to avoid degradation and increased optical loss. In this context, different techniques can be applied to protect them. One possible approach is to embed the tapered fiber in polymers such as Teflon, which allows to protect and stabilize it [16]. However, the optical attenuation is significantly increased under this approach [17]. Another solution is to use silica aerogel, which consists of nanostructured silicon dioxide $\left(\mathrm{SiO}_{2}\right)$ with a density of 0.1 grams per cubic centimeter $[17,18]$. In the aerogel, the pores and silica network are structured in the scale of tens of nanometers, which is much smaller than visible and infrared light wavelengths. Moreover, aerogel is permeable to gas and has a refractive index very close to the one of the air. Thus, silica aerogels are useful to protect fiber tapers without disturbing light propagation through it [17] and can act, together with fiber tapers, in gas sensing setups [19]. Nevertheless, silica aerogel preparation is not straightforward as it involves mastering its chemical synthesis process [20].

In the latest years, the 3-dimensional (3D) printing technology has appeared as a powerful methodology able to be applied in the fiber optics domain. For example, it has been demonstrated that optical fibers can be directly drawn from 3D printed preforms [20-24]. Additionally, 3D printing technology can be used for creating casings in which optical fibers can be embedded. Sensors based on fiber Bragg gratings (FBGs), for example, have been reported to benefit from this approach [25-27]. However, to the best of our knowledge, there is no report on the application of $3 \mathrm{D}$ printed casings to protect fiber tapers.

In this framework, for the first time, we propose the use of $3 \mathrm{D}$ printing technology for creating housings for fiber tapers (taper cages). Under this approach, we show that the taper cages can be used to protect the waveguide against humidity while keeping the possibility of gas diffusion, as required for gas sensing. The cages were built by using a low-cost desktop 3D printer according to a layer-by-layer routine. We experimentally show that, by adequately tailoring the cage meshing, different performances regarding taper protection to humidity can be obtained. Moreover, we demonstrate the possibility of acetylene $\left(\mathrm{C}_{2} \mathrm{H}_{2}\right)$ detection. We envisage that this new methodology will provide novel opportunities in the design of tapered fibers devices with increased resistance to humidity deterioration and is able to perform gas sensing activities.

\section{Device fabrication}

The taper cages were fabricated from a 
1.75-mm-thick commercial polylactic acid (PLA) filament via the utilization of a desktop 3D printer (Sethi3D AiP). Their fabrication followed a procedure that involved $3 \mathrm{D}$ printing and manual accommodation of the taper inside the cage. In the first step (Step \#1 in Fig. 1), the bottom part of the taper cage was created. The external dimensions of the $3 \mathrm{D}$ printed sample were set to be $13 \mathrm{~mm} \times$ $13 \mathrm{~mm} \times 130 \mathrm{~mm}$. At the cage extremities, two slots were made (with dimensions $1 \mathrm{~mm} \times 1 \mathrm{~mm} \times 5 \mathrm{~mm}$ ) for taper positioning. In the central region of the cage, a broader channel (with dimensions $7 \mathrm{~mm} \times 7$ $\mathrm{mm} \times 30 \mathrm{~mm}$ ) was created for housing the taper waist. During the printing procedure, the printing board was kept at $50{ }^{\circ} \mathrm{C}$ to enhance the sample adhesion to it. In sequence, a tapered optical fiber (fabricated from a standard telecom single-mode fiber, SMF-28, and by using the flame brushing technique) was manually transferred from the taper rig to the printed sample (Step \#2 in Fig. 1). In this process, the fiber taper was dragged by one of its extremities and carefully positioned on the slots at the cage edges. The untapered region of the fiber was then glued to the cage slots by using cyanoacrylate glue. Care was taken to keep the taper waist and the transition regions suspended in air while the untapered fiber sections were supported by the narrow slots of the cage.

In the third step (Step \#3 in Fig. 1), the cage cover was separately printed and manually glued to the bottom part also by using the cyanoacrylate glue. Here, it is worth saying that one has also tried to directly resume the printing routine after placing the fiber taper on the bottom part of the cage. Although efficient in terms of fabrication, this procedure added much loss to the taper since polymer debris happened to be accidentally deposited on the fiber taper during the cage cover printing. The gluing procedure, therefore, was found to be the most successful method to preserve the taper optical transmission quality. However, further improvements in the fabrication process (such as fine-tuning of the extruder temperature) would potentially allow the realization of direct fabrication of the taper cage without the need for glue application.

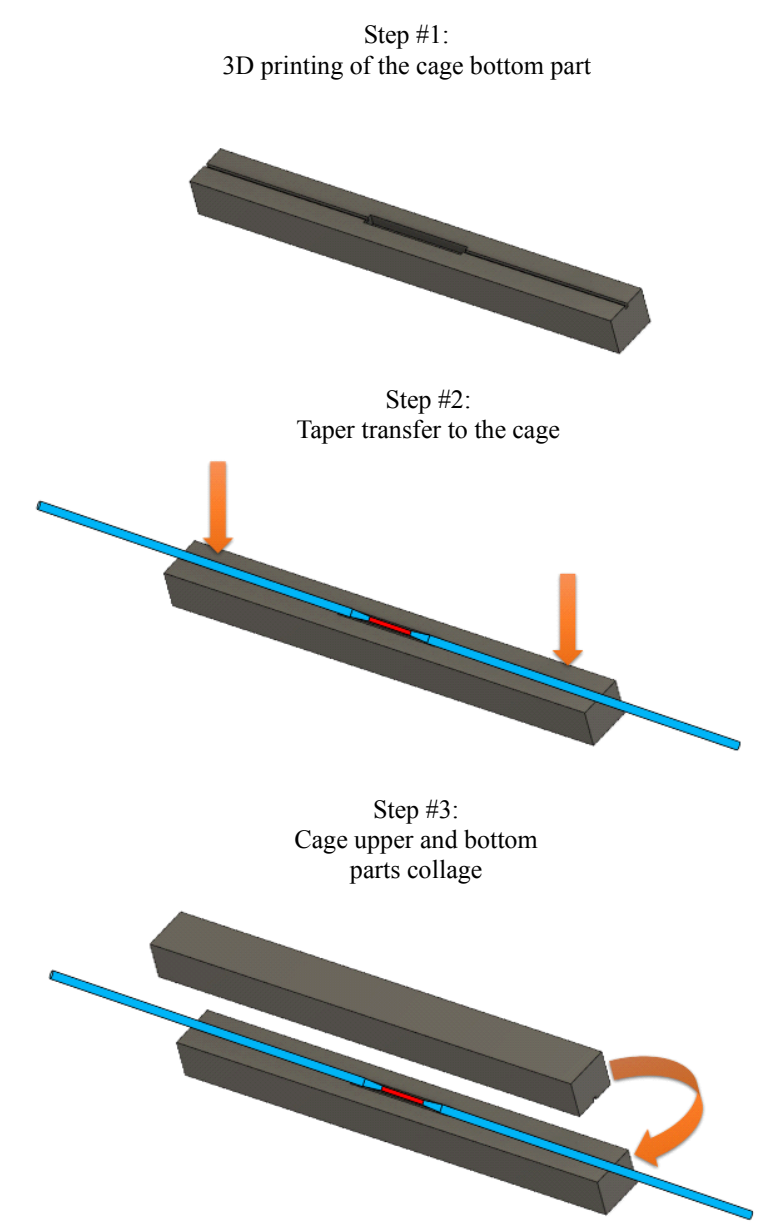

Fig. 1 Schematic diagram of the taper cage preparation procedure.

To characterize the increment of the taper optical loss due to the cage setting-up procedure, the microfiber optical response was measured as a function of the time while the cage preparation was being conducted. In the experiment, a super-LED (SLED, centered at $1550 \mathrm{~nm}$ ) was connected to the fiber input, and a power meter measured the transmitted signal. Figure 2 presents the measured data, which stands for the procedure described in the following. The tapering process started at $0 \mathrm{~s}$ and finished at $300 \mathrm{~s}$. Between $350 \mathrm{~s}$ and $700 \mathrm{~s}$, the taper was transferred from the taper rig to the cage bottom part, and the cover part was cast on the fiber. The 
procedure was finished around $700 \mathrm{~s}$ when a loss value of $0.53 \mathrm{~dB}$ could be measured. Note that there was a slight decrease in the taper loss when comparing the end of the fabrication process $(\sim 300 \mathrm{~s})$ and the final assembling moment $(\sim 700 \mathrm{~s})$ due to the application of a small strain level to the taper to make it straight. The procedure of the cage setting-up, therefore, added minimum optical loss to the fiber taper sample. Indeed, adequate fiber taper handling and care during cage preparation procedure allow replicating fiber cage preparation with a typical loss of around $0.5 \mathrm{~dB}$.

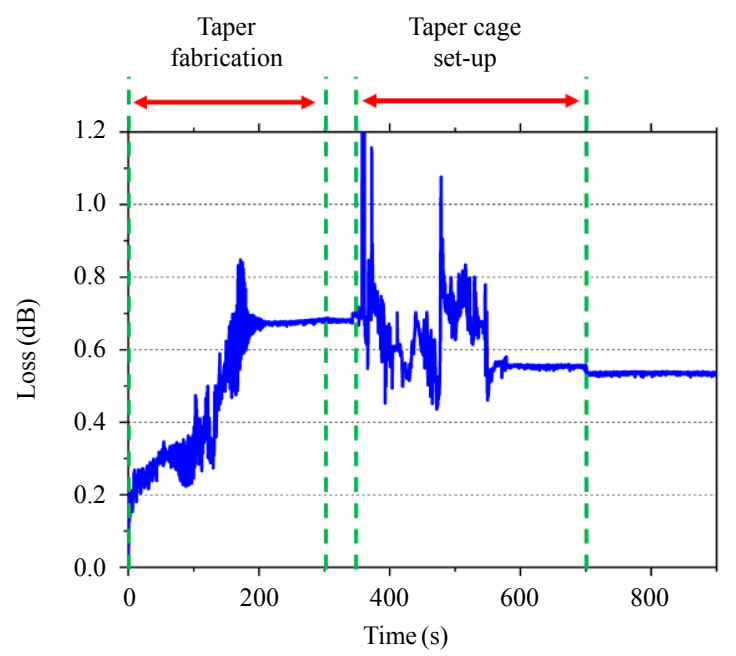

Fig. 2 Optical loss as a function of the time (taper waist diameter: $1 \mu \mathrm{m}$; taper waist length: $10 \mathrm{~mm}$ ). Taper fabrication happens between $0 \mathrm{~s}$ and $300 \mathrm{~s}$ and taper cage setting-up takes place between $350 \mathrm{~s}$ and $700 \mathrm{~s}$.

\section{Humidity protection and gas detection}

\subsection{Taper cages for humidity protection}

Three different samples were prepared to test the proposed taper cages for humidity protection. In each sample, different meshing was employed during the cage upper part fabrication. It entailed distinct material filling densities in each sample (ratio between the volume of the plastic material used in the upper part of the cage and the whole volume of the latter). Figure 3 shows pictures of the three samples we employed herein, each one with different material filling densities, namely $30 \%$, $50 \%$, and $70 \%$. In all samples, the bottom part of the cage had the same fabrication parameters. Also, the fiber tapers parameters were the same in all tested samples $(1 \mu \mathrm{m}$ diameter and $10 \mathrm{~mm}$ length). (a)

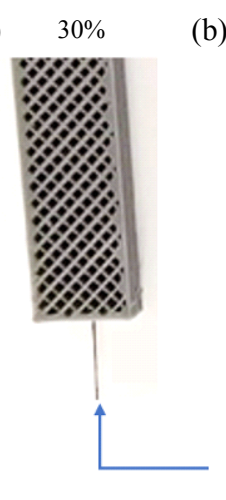

(b)

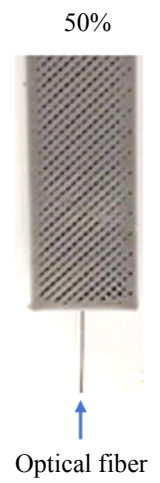

(c) $\quad 70 \%$

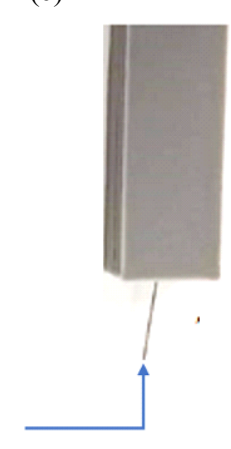

Fig. 3 Pictures of the taper cages with (a) $30 \%$, (b) $50 \%$, and (c) $70 \%$ material filling densities.

The taper cages were placed inside a chamber (with an approximate volume of $45 \mathrm{~cm}^{3}$ ) to check the humidity protection potential of the proposed technique. A hygrometer and a portable ultrasonic humidifier were also included. In the experiment, the taper optical loss was monitored as a function of the time while the humidity generator was sequentially turned on and off.

Figure 4 shows the results of the optical loss as a function of the time for tapers inside cages with material filling densities of $30 \%, 50 \%$, and $70 \%$ (purple, blue, and green lines, respectively). Additionally, Figure 4 shows the loss of a bare fiber taper as a function of the time. Data in Fig. 4 refer to the procedure described in the following. The portable humidifier was maintained turned off for $100 \mathrm{~s}$ and then turned on for $50 \mathrm{~s}$. This cycle was, then, sequentially repeated (periods of humidification in the ON state are represented in Fig. 4). The relative humidity was measured to be between $90 \%$ and $95 \%$ while the humidifier was maintained turned on. For the periods in which the humidifier was kept turned off, a decrease in the relative humidity values was measured, reaching values between $75 \%$ and $80 \%$. Additionally, it is worth mentioning that, after turning on the humidifier, water condensation has been observed inside the chamber where the taper cages were 
tested.

The black curve in Fig. 4 represents the bare taper time response. It is seen that the optical loss increased to $24 \mathrm{~dB}$ as soon as the humidifier was turned on (at $50 \mathrm{~s}$ ). Moreover, one can observe that this loss level was maintained up to the end of the experiment (even in the periods when the humidifier was set to off mode).

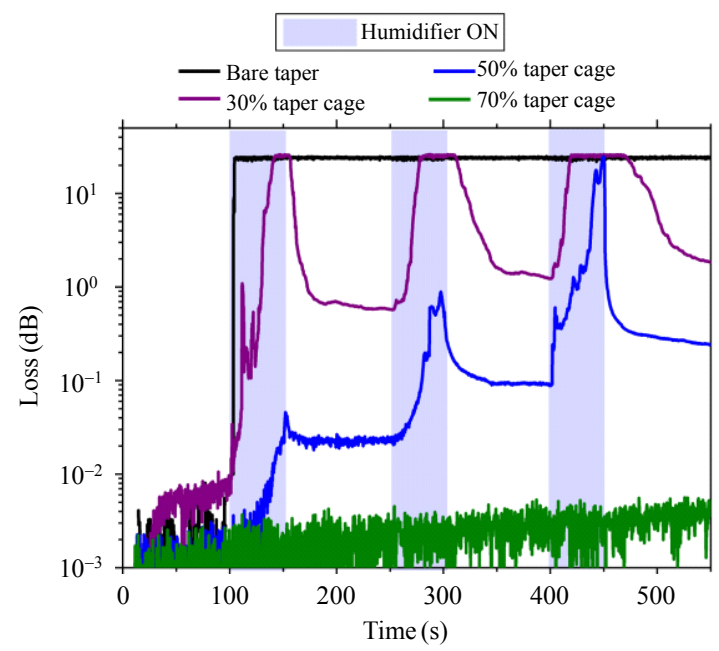

Fig. 4 Time evolution of the optical loss of tapered fibers ( $1 \mu \mathrm{m}$ diameter) embedded in 3D printed cages with $30 \%$ (purple curve), $50 \%$ (blue curve), and 70\% (green curve) material filling densities. Data for the bare fiber taper are shown in black.

For the case in which the $30 \%$ filling density cage was tested (purple line in Fig.4), an increase in the taper loss was also observed. However, it was not as immediate as in the bare taper case. For the taper in the $30 \%$ material filling density cage, the loss started growing around $30 \mathrm{~s}$ after the humidifier has been turned on. Additionally, it is remarkable that, differently than the bare fiber case, the taper loss was dramatically reduced during the periods in which the humidifier was maintained turned off. One possible explanation for this observation would be to assume that the lower optical loss of the taper in the cage was generated by a lower amount of water than that in the bare taper case (since the cage prevents part of the water molecules from achieving to the fiber taper). This lower amount of water would be, therefore, more easily detached from the fiber taper via evaporation during the periods with lower relative humidity. Moreover, it should be noted that, although low loss figures were measured during the periods in which the humidifier was off, they have slightly grown as a function of the time $(0.02 \mathrm{~dB}$ at $200 \mathrm{~s} ; 0.09 \mathrm{~dB}$ at $375 \mathrm{~s}$ and $0.24 \mathrm{~dB}$ at $550 \mathrm{~s})$. It can be explained by the accumulation of water molecules on the taper during the on-off humidity cycles.

The blue line in Fig. 4 shows the loss of the taper in the $50 \%$ filling density cage as a function of the time. For this one, loss values higher than $0.1 \mathrm{~dB}$ were only seen in the second humidity cycle. As expected, an increase in the material density in the cage meshing entailed improved protection to humidity. In the third humidity cycle, the loss reached $25 \mathrm{~dB}$. However, it strongly dropped after the humidifier was turned off. At $550 \mathrm{~s}$, the loss level was measured to be $0.24 \mathrm{~dB}$.

Finally, one tested the taper in the $70 \%$ material filling density cage. For this sample, very low loss values $(<0.01 \mathrm{~dB})$ were measured in the whole experiment (green line in Fig. 4). Indeed, in all tests using the $70 \%$ filling density cage, the taper loss was inferior to $0.01 \mathrm{~dB}$, which indicates that the results are robust and consistent. One can conclude, therefore, that embedding the fiber taper in the 3D-printed taper cage is an efficient approach to avoid taper signal deterioration due to humidity.

\subsection{Gas detection}

As described in the last section, the use of taper cages avoids taper loss deterioration due to humidity. As an additional feature, since the cage is permeable to gas diffusion due to its tailorable meshing, these structures can be used in gas detection experiments. It would not be the case, for example, if a fully closed box was used to protect the tapered fiber. To illustrate this operation, we prepared three taper cages with material filling densities of $30 \%, 50 \%$, and $90 \%$. In all cages, the taper samples were again $1-\mu \mathrm{m}$ thick and $10-\mathrm{mm}$ long. 
For the gas detection measurements, the taper cages were inserted in a gas chamber where acetylene $\left(\mathrm{C}_{2} \mathrm{H}_{2}\right)$ could be injected. A manometer allowed the verification of the acetylene pressure inside the chamber. A SLED with emission centered at $1550 \mathrm{~nm}$ was used as the light source, and an optical spectrum analyzer (OSA) accounted for the transmitted signal. By observing the transmitted spectrum, acetylene absorption lines could be seen, and gas detection measurements could be performed.

Figure 5 presents the normalized transmitted power as a function of the wavelength for the different taper cages when subjected to an acetylene pressure of 2 bar. The normalized power values were calculated by comparing the transmitted spectra before and after acetylene injection in the chamber. Data in Fig. 5 show that the acetylene absorption lines could be observed when measuring the fiber taper transmission in all samples. The experiment was performed at room temperature, and no considerable temperature variations took place during the measurements.

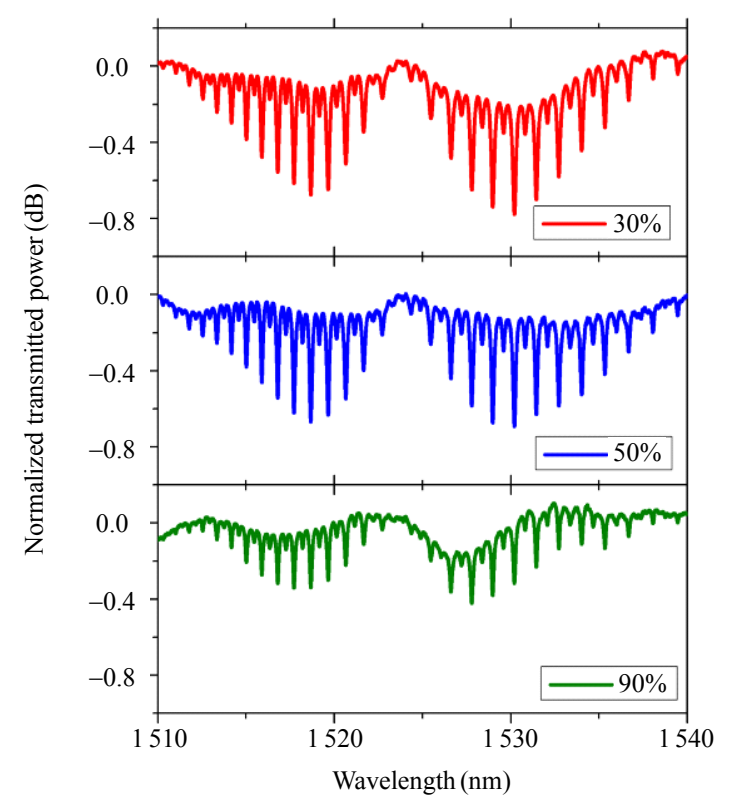

Fig. 5 Normalized transmitted power for the tapered fibers inside the taper cages (with material filling densities of $30 \%$, $50 \%$, and $90 \%$ ).

In the experiments, the maximum contrast of the absorption lines was measured to be $0.56 \mathrm{~dB}$. It is noteworthy, however, that the contrast of the absorption lines is smaller for the taper inside the cage with $90 \%$ material filling density. It is expected because the denser meshing in the $90 \%$ material filling density taper cage entails diminished acetylene diffusion through the cage. Here, it should be underlined that the spectra in Fig. 5 can be compared since they were accounted at similar time intervals between the moment when acetylene was inserted in the chamber and the moment of the spectral measurement itself. It is an essential aspect because, in our measurements, the device response time was seen to be dependent on the material filling density of the taper cage (due to the different gas diffusion rates in cages with distinct meshing densities). The results allow observing that the utilization of the proposed cages keeps the possibility of using fiber tapers as gas detection devices.

\section{Conclusions}

In this paper, we propose the utilization of 3D printing technology for the protection of optical fiber tapers. To the best of our knowledge, this is the first time that $3 \mathrm{D}$ printed cages were used to prevent taper optical transmission deterioration due to humidity. The method used herein is more straightforward than other taper protection techniques because it avoids the need for chemical processes as it is wholly based on the production of taper cages in a commercial 3D printer.

Here, we showed that the taper cage preparation added very low loss to the fiber taper sample $(0.5 \mathrm{~dB})$. Moreover, we analyzed taper cages with different meshing and demonstrated that its taper protection ability could be tailored by suitably choosing the material filling densities in the cage. Additionally, we performed gas detection experiments to illustrate the potential of the application of the 3D-printed taper cages for sensing applications. 
We understand that this novel approach provides a new avenue for the design of fiber taper-based devices able to better resist in humid environments while keeping their gas sensing capabilities.

\section{Acknowledgment}

This research was supported by Fundação de Amparo à Pesquisa do Estado de São Paulo (FAPESP) (Grant No. 2017/06411-3).

Open Access This article is distributed under the terms of the Creative Commons Attribution 4.0 International License (http://creativecommons.org/licenses/by/4.0/), which permits unrestricted use, distribution, and reproduction in any medium, provided you give appropriate credit to the original author(s) and the source, provide a link to the Creative Commons license, and indicate if changes were made.

\section{References}

[1] S. Pevec and D. Donlagic, "Multiparameter fiber-optic sensors: a review," Optical Engineering, 2019, 58(7): 072009.

[2] P. Russell, "Photonic crystal fibers," Science, 2003, 299(5605): 358-362.

[3] V. Portosi, D. Laneve, M. C. Faloni, and F. Prudenzano, "Advances on photonic crystal fiber sensors and applications," Sensors, 2019, 19(8): 1892.

[4] S. Silva, E. G. P. Pachon, M. A. R. Franco, J. G. Hayashi, F. X. Malcata, O. Frazão, et al., "Ultrahigh-sensitivity temperature fiber sensor based on multimode interference," Applied Optics, 2012, 51(16): 3236-3242.

[5] A. D. Kersey, M. A. Davis, H. H. Patrick, M. LeBlanc, K. P. Koo, C. G. Askins, et al., "Fiber grating sensors," Journal of Lightwave Technology, 1997, 15(8): 1442-1463.

[6] J. Lou, Y. Wang, and L. Tong, "Microfiber optical sensors: a review," Sensors, 2014, 14(4): 5823-5844.

[7] G. Brambilla, "Optical fibre nanowires and microwires: a review," Journal of Optics, 2010, 12(4): 043001.

[8] R. Yang, Y. Yu, Y. Xue, C. Chen, Q. Chen, and H. Sun, "Single S-tapered fiber Mach-Zehnder interferometers," Optics Letters, 2011, 36(23): 4482-4484.

[9] N. Vukovic, N. G. R. Broderick, M. Petrovich, and G. Brambilla, "Novel method for the fabrication of long optical fiber tapers," IEEE Photonics
Technology Letters, 2008, 20(14): 1264-1266.

[10] J. M. Ward, D. G. O’Shea, B. J. Shortt, M. J. Morrissey, K. Deasy, and S. G. N. Chormaic, "Heat-and-pull rig for fiber taper fabrication," Review of Scientific Instruments, 2006, 77(8): 083105.

[11] T. A. Birks and Y. W. Li, "The shape of fiber tapers," Journal of Lightwave Technology, 1992, 10(4): 432-438.

[12] C. M. B. Cordeiro, W. J. Wadsworth, T. A. Birks, and P. St. J. Russell, "Engineering the dispersion of tapered fibers for supercontinuum generation with a 1064 nm pump laser," Optics Letters, 2005, 30(15): 1980-1982.

[13] F. Beltrán-Mejía, J. H. Osório, C. R. Biazoli, and C. M. B. Cordeiro, "D-microfibers," Journal of Lightwave Technology, 2013, 31(16): 3056-3061.

[14] W. Jin, H. L. Ho, Y. C. Cao, J. Ju, and L. F. Qi, “Gas detection with micro- and nano-engineered optical fibers," Optical Fiber Technology, 2013, 19(6): 741-759.

[15] G. Brambilla and D. N. Payne, "The ultimate strength of glass silica nanowires," Nano Letters, 2009, 9(2): 831-835.

[16] N. Lou, R. Jha, J. L. Domínguez-Juárez, V. Finazzi, J. Villatoro, G. Badenes, et al., "Embedded optical micro/nano-fibers for stable devices," Optics Letters, 2010, 35(4): 571-573.

[17] L. Xiao, M. D. W. Grogan, W. J. Wadsworth, R. England, and T. A. Birks, "Stable low-loss optical nanofibers embedded in hydrophobic aerogel," Optics Express, 2011, 19(2): 764-769.

[18] L. Tong, J. Lou, R. R. Gattass, S. He, X. Chen, L. Liu, et al., "Assembly of silica nanowires on silica aerogels for microphotonic devices," Nano Letters, 2005, 5(2): 259-262.

[19] L. Xiao, M. D. W. Grogan, R. England, W. J. Wadsworth, and T. A. Birks, "Gas sensing with a sub-micron tapered fibre embedded in hydrophobic aerogel," in Conference on Lasers and Electro-Optics 2010, California, May 16-21, 2010, pp. 1-3.

[20] J. L. Gurav, I. Jung, H. Park, E. S. Kang, and D. Y. Nadargi, "Silica aerogel: synthesis and applications," Journal of Nanomaterials, 2010, 2010: 409310.

[21] K. Cook, J. Canning, S. Leon-Saval, Z. Reid, M. A. Hossain, J. Comatti, et al., "Air- structured optical fiber drawn from a 3D-printed preform," Optics Letters, 2015, 40(17): 3966-3969.

[22] K. Cook, G. Balle, J. Canning, L. Chartier, T. Athanaze, M. A. Hossain, et al., "Step-index optical fiber drawn from 3D printed preforms," Optics Letters, 2016, 41(19): 4554-4557.

[23] T. H. R. Marques, B. M. Lima, J. H. Osório, L. E. da Silva, and C. M. B. Cordeiro, "3D printed microstructured optical fibers," in 2017 SBMO/IEEE MTT-S International Microwave and 
Optoelectronics Conference (IMOC), Brazil, Aug. 27-30, 2017, pp: 1-3.

[24] W. Talataisong, R. Ismaeel, T. H. R. Marques, S. A. Mousavi, M. Beresna, M. A. Gouveia, et al., "Mid-IR hollow-core microstructured fiber drawn from a 3D printed PETG preform," Scientific Reports, 2018, 8(1): 8113.

[25] M. G. Zubel, K. Sugden, D. J. Webb, D. Saez-Rodriguez, K. Nielsen, and O. Bang, "Embedding silica and polymer fibre Bragg gratings (FBG) in plastic 3D-printed sensing patches,"
Micro-structured and Specialty Optical Fibres IV, 2016, 9886: 98860N.

[26] N. R. Manzo, G. T. Callado, C. M. B. Cordeiro, and L. C. M. Vieira Jr., "Embedding optical fiber Bragg grating (FBG) sensors in 3D printed casings," Optical Fiber Technology, 2019, 53: 102015.

[27] R. Scott, M. Vidakovic, S. Chikermane, B. McKinley, T. Sun, P. Banerji, et al., "Encapsulation of fiber optic sensors in 3D printed packages for use in civil engineering applications: a preliminary study," Sensors, 2019, 19(7): 1689. 